\title{
The research progress of application of sequencing technology to analyze the genetic information of malignant tumor, especially drug resistant recurrent ovarian cancer
}

\author{
Zhenhua Du* and Qing Yang \\ Department of Obstetrics and Gynecology, Shengjing hospital affiliated to China medical university, Shenyang, Liaoning, China
}

\begin{abstract}
The second-generation sequencing (NGS) technology plays an important role in the research of contemporary tumor research. It provides a fast and accurate method for the detection of tumor mutant genes and drug resistance genes. ctDNA is DNA derived from tumor cells and can accurately reflect tumor genome changes in real time. It also plays an indispensable role in all fields of cancer research. This article will elaborate and summarize the application of ctDNA, the current application of NGS detection and the detection of ctDNA changes in ovarian cancer resistance and recurrence.
\end{abstract}

\section{Introduction}

Ovarian cancer is a malignant tumor that seriously threatens the life safety of women, and its fatality rate is the first in female genital malignant tumors. Because of the early clinical symptoms and signs are relatively hidden and not easy to detect, and due to the lack of effective screening methods, frequently, most of the $70 \%$ of the patients are late at the time of medical treatment, accompanied by pelvic and abdominal metastases optically. At present, the principle of treatment for advanced ovarian cancer is operation, combined with chemotherapy, radiotherapy and other comprehensive treatment, of which platinum based chemotherapy is a major adjuvant therapy for advanced ovarian cancer. But even as the most commonly used firstline chemotherapy drug, its primary or secondary drug resistance is a major obstacle to the treatment of ovarian cancer, it is also one of the main reasons that the five - year survival rate of patients with advanced ovarian cancer is difficult to exceed $30 \%$. After the initial treatment of ovarian cancer patients, about $70 \%-80 \%$ of the patients have a recurrence. The recurrence of ovarian cancer is defined as a relatively short duration of chemotherapy, which occurs within 6 months. However, for patients with drug-resistant recurrent ovarian cancer, platinum based chemotherapy cannot be continued, the choice of drugs is less, the treatment is difficult, the survival period is short, and the prognosis is poor. Therefore, to find out the causes of platinum resistance in ovarian cancer is the problem that we need to solve urgently. It is also the precondition for rational selection of effective drugs for ovarian cancer. In recent years, studies have shown that the heterogeneity of tumor plays a very important role in the drug resistance of ovarian cancer [1].

Tumor heterogeneity is an important manifestation of tumor recurrence and metastasis, and it is also the main restriction of tissue biopsy [2,3]. The heterogeneity of tumor shows that the same tumor shows different genetic characteristics in different regions(namely, intratumoral heterogeneity). Similarly, heterogeneity exists in metastatic tumor of the same patient (namely, transfer heterogeneity)
[1]. Because the tumor is heterogeneous, so the pathological tissue obtained by surgery or biopsy can only reveal the genetic information of tumor unit point and cannot fully reveal the biological characteristics and dynamic changes of tumor, and then it will affect the selection of the clinical treatment plan and the accuracy of the evaluation of the curative effect. Circulating tumor DNA (ctDNA), also known as liquid biopsy, can remedy the defect of tissue biopsy, it reflects the changes of blood circulation in patients with tumor genomic information rather than unit information, break through the tumor heterogeneity constraints, it provides technical possibility for real-time and dynamic monitoring of tumor whole genome information [4].

\section{The characteristics and application of ctDNA}

Although tumor tissue is the gold standard for clinical and research sequencing, it is still difficult to obtain and apply the tissue. From the perspective of sampling, biopsy is not a simple method. In addition, biopsy increases the cost of nursing and makes the patient feel sick, but this invasive examination will not affect the result. The last and most important point is that a biopsy may have a clinical complication. The MD Anderson cancer center report showed that the incidence of adverse events in trachea biopsy and abdominal and pelvic biopsy was $17.1 \%$ and $1.6 \%$, respectively [5].

In addition to the problem of tissue acquisition, the use of tumor tissue for gene sequencing is also affected by the problem of sample preservation and the problem of tissue heterogeneity. Most of the

${ }^{\star}$ Correspondence to: Zhenhua Du, Department of Obstetrics and Gynecology, Shengjing hospital affiliated to China medical university, Shenyang, Liaoning110021, China,duzhenhua0000@163.com

Key words: ctDNA, second-generation sequencing NGS, ovarian cancer, drug resistance relapse

Received: October 23, 2018; Accepted: November 08, 2018; Published: November 12, 2018 
tumor tissues are preserved in formalin fixed paraffin embedded blocks and are cross-linked with DNA. In some cases, the preserved samples cannot be adequately analyzed in molecular analysis. Although this approach has been improved in single gene mutations and target gene sets, there are still limitations in the full genome exon analysis. In addition, the number of cancer cells in each biopsy is different, largely influenced by the density of the tumor cells (the percentage of the tumor) and the size of the sample required, the number of cancer cells is also affected by the number of tissues in fine needle aspiration or thick needle biopsy. Compared with the samples removed during operation, the number of tumor tissue obtained by puncture is small.

Biopsy or partial tissue section of a single tumor will lose heterogeneity and metastasis in the tumor. In theory, circulating tumor DNA (ctDNA) can provide the same genetic information as tissue biopsy. CtDNA is a class of double stranded DNA fragments with a size of $0.18 \sim 21 \mathrm{~kb}$. They originate from tumor cells. They mainly exist in the blood of tumor patients, synovial fluid and cerebrospinal fluid, and can be excreted by patients' urine and feces. CtDNA, also known as "liquid biopsy", can reflect the whole picture of tumor genome information, so it can break through the restriction of tumor heterogeneity. On the other hand, it is a source of fresh DNA, not affected by preservatives. Using a minimally invasive technique, a needle tube is used to sample blood to avoid the risk of biopsy. In addition, during the period of treatment, blood can be extracted at any time to dynamically monitor the molecular changes in the tumor [6-8]. The ctDNA fragment contains the same genetic defects as the tumor itself. These DNA changes include all types of genomic alterations in the tumor, such as point mutations (EGFR and KRAS), rearrangement (EML4-ALK), gene proliferation (HER2 and MET) and aneuploidy [1].

As a dynamic marker of tumor, ctDNA has more advantages than traditional protein markers and imaging examinations. The half-life period of ctDNA is short (about 2 hours), so it can respond to a few hours of tumor changes rather than weeks or months [9]. Therefore, the change of ctDNA is a few weeks or even a few months earlier than the imaging examination or the protein marker [10]. Furthermore, protein markers, such as CA125, are not only elevated in patients with malignant tumors, but also in benign tumors and inflammation. Another feature of ctDNA is highly sensitive. Studies have shown that the sensitivity of liquid biopsies in patients with stage IV tumors is nearly $100 \%$ [11]. Although the sensitivity of ctDNA is high in advanced cancer, it must be clear that the sensitivity of ctDNA is mainly influenced by biological and technical factors. When there is a low-grade tumor or a small metastasis, the content of ctDNA is also reduced. In strict sense, the sensitivity of ctDNA detection is also limited by the DNA polymerase error rate (usually $0.01 \%$ ). Therefore, if the error rate of the ctDNA fragment in the sample is less than or equal to $0.01 \%$, then the ctDNA can be considered negative. Another important advantage of ctDNA analysis is highly specific, which can be used to detect tumor specific molecular changes in the blood circulation. Because the ctDNA mutation is targeted to the tumor individual and exists in the tumor DNA, it does not appear in the normal cell DNA. Because of its high sensitivity and specificity, ctDNA can be used for molecular heterogeneity assessment, monitoring tumor dynamics, identifying gene therapy factors, tracking the evolution of genome and development of drug resistance. Therefore, it has a broad application prospect in exploring the molecular mechanism of tumor recurrence and metastasis and drug resistance [1].

\section{Clinical application of using NGS to detect ctDNA in tumor}

The application of high-throughput gene sequencing (NGS) detection technology can detect ctDNA content in body fluid, so that we can get driving gene information for tumor mutation and cancer cell specific antigen, and then discover a new generation of biomarkers. With the research of tumor genome, more and more tumor related genome information has been discovered. Now we know that almost all kinds of cancers are related to gene mutation in somatic cells. These types of mutation include single base replacement, insertion mutation, deletion mutation and chromosome translocation (gene fusion, gene amplification, loss of heterozygosity). In the whole cell population, the mutation frequency of these somatic cells is very low, so it can be used as a specific biomarker from a biological perspective [12].

\section{Early diagnosis}

In a Bettegowda study, gene mutations could be detect from all 410 cancer patients, which made ctDNA a further biomarker for cancer patients. In addition, the level of ctDNA detected in over $80 \%$ of metastatic tumor patients is much higher than other traditional biomarkers, such as protein markers CA125, CEA and so on. Unlike protein markers, gene mutation occurs only in tumor cells, but protein markers are not only found in tumor cells, but also in normal cells [13].

A recent study showed that plasma ctDNA integrity in the value of early diagnosis of breast cancer, the concentration of ctDNA combined with CFDI (cell-free integrity) has important value in early diagnosis of primary breast cancer (PBC) and metastatic breast cancer (MBC) . The study was carried out in 383 patients with breast cancer, including 82 cases of PBC and 201 cases of MBC and 100 healthy controls. The results showed that ctDNA concentration combined with CFDI can well distinguish primary breast cancer, metastatic breast cancer and healthy controls [14].

In 2013, the study of colorectal cancer patients by Da and other scholars showed that the value of plasma CFDI was of great significance in the diagnosis of colorectal cancer. The study included 27 cases of non-operation treatment for patients with colorectal cancer, 33 cases of patients with colorectal cancer and 33 healthy controls, the results showed that the differences of CFDI value of plasma in operation group and non-operation group, operation group and health control group, non-operation group and healthy control group were all of statistical significance [15].

In addition, Leszinski, et al. [16] showed that in the diagnosis of colon cancer, the integrity of ctDNA is valuable in the results of the 2014 study.

\section{Monitor tumor burden and determine prognosis}

CtDNA can also monitor the changes of tumor load in real time, dynamically and noninvasive, so it can be used as a new biomarker in clinical research. The study results of Luis, et al. show that the detection of ctDNA in metastatic tumor patients can guide the clinical treatment, can monitor the recurrence of the tumor and suggest the prognosis. For example, the low level of ctDNA in patients with metastatic colorectal cancer (CRC) indicates a good prognosis. This conclusion indicates a correlation between ctDNA concentration and survival rate. The same conclusion also appears in the study of patients with advanced breast cancer [1]. In addition, in a case-control study of gastric cancer, the researchers compared the changes of plasma ctDNA levels before and after operation. The results showed that plasma ctDNA levels were significantly lower than those before operation. Therefore, it is concluded that ctDNA quantification can be used to evaluate the completeness of surgical resection for tumor patients, that is, the more complete surgical resection is, the lower the ctDNA content, and the ctDNA quantification can also prompt the metastasis and recurrence of the tumor very early [17]. 
In addition, many studies have shown that ctDNA can also be used to judge the prognosis of cancer patients with high accuracy. A study by Lecomte, et al. explored the value of the K-ras gene mutation in the peripheral blood ctDNA and hypermethylation of the CDKN2A promoter in the prognosis of colorectal cancer, in this study, 37 patients with colorectal cancer were followed up for an average of 22 months and the survival analysis was performed. The results showed that the 2 year overall survival rate $(48 \%, 95 \%$ CI $26 \% \sim 66 \%)$ of K-ras gene mutation and CDKN2A promoter hypermethylation was significantly lower than the overall survival rate (100\%) of patients without the above gene mutation. COX regression analysis showed that ctDNA K-ras gene mutation and hypermethylation of CDKN2A promoter was an independent prognostic factor of CRC. This result further illustrates the important value of K-ras gene mutation or CDKN2A promoter hypermethylation in the prognosis of CRC patients, and is also expected to become a biomarker for predicting the recurrence risk of CRC patients. In a study published in 2012, a high level of K-ras gene mutation was found to be associated with a poor prognosis of CRC. In this study, 108 patients with metastatic CRC were treated with cetuximab combined with irinotecan, and the levels of $\mathrm{K}$-ras and BRAF mutations in plasma ctDNA were quantitatively detected before treatment. The results showed that the disease control rate of patients with K-ras mutation level above $75 \%$ was significantly lower than that of the low-level mutation patients $(\mathrm{P}<0.048)$. Cox regression analysis showed that the mutation level of K-ras gene in ctDNA was significantly correlated with the prognosis of metastatic CRC. The prognosis of CTC patients with a high level of K-ras gene mutation is poor [18]. A similar report was also reported in a study on changes in plasma ctDNA in patients with metastatic breast cancer in 2013 by Dawson, et al. [19].

\section{Evaluation of efficacy and detection of drug resistance in time}

In recent years, many scholars are committed to the study of monitoring ctDNA specific gene alterations in peripheral body fluid, such as gene mutation types and copy number variations, so as to achieve the purpose of curative effect evaluation. For example, in a study of metastatic breast cancer, the results showed that ctDNA could reflect disease progression and therapeutic effect earlier than circulating tumor cells, carbohydrate antigen 153 (CA153) and imaging examination [19]. In another study of metastatic breast cancer and ovarian cancer, plasma samples were used to track the dynamic changes of tumor specific mutation genes. The results showed that ctDNA could reflect the clinical course of disease [20].

Some patients with tumor targeted therapy can acquire acquired resistance. For example, in a study by Diaz, et al. [21] the ctDNA was esed to monitor the related mutation genes and evaluate the efficacy of targeted therapy. The researchers found that for K-ras wild type patients with metastatic CRC, some of them still have some resistance after targeted therapy with panitumumab. After testing the ctDNA of these patients, it was found that the K-ras gene mutation appeared in the form of low frequency subclones before treatment, and at a followup of 5 to 6 months later, $38 \%$ of the K-ras mutation was found in the ctDNA of the population, and a new mutation of the BRAF gene was found in 9 patients. The results showed that by monitoring the changes in gene spectrum of ctDNA targeted therapy, we could evaluate the efficacy of targeted therapy, and adjust the selection of drugs based on this basis in clinical treatment. And at present, compare the body fluid ctDNA to the tumor tissue DNA, the sensitivity, specificity and consistency of the detection of specific gene changes are still lacking a unified conclusion and need to be further verified by clinical data. The research of Hickey, et al. have detected ctDNA in serum and ascites of ovarian cancer patients. The results show that some molecular variants are closely related to the clinical stage, curative effect evaluation and prognosis of patients. Song $\mathrm{Xu}$, et al. reported that using NGS to detect ctDNA in tissue samples and matched blood samples in advanced lung cancer, the agreement rate could reach 76.2\% [22]. Evelyn Kidess, et al. [23] detected 46 genes in colorectal cancer patients and compared the consistency of four genes such as KRAS/PIK3CA/BRAF/EGFR. The agreement rate of 19 patients with stage IV was $93 \%$, and the agreement rate of 19 patients with stage I-III reached 54 . \%, however, JiaCheng Tang, et al. [24] reported that gene detection of tissue DNA and humoral ctDNA in liver cancer patients showed that the specificity, sensitivity, and consistency of the samples were very different.

\section{Analysis of genetic biological information of patients with drug-resistant recurrent ovarian cancer}

The recurrence of drug-resistant ovarian cancer may be caused by a variety of factors, including changes in the patient's own and the changes in the gene of the tumor cells. This is the most serious problem in the treatment of cancer. The high frequency of cancer cell resistance suggests the occurrence of special molecular mechanisms in the tumor. Oncogenes are abnormal forms of proto-oncogenes during cell growth and differentiation. When activated by specific point mutations, chromosome shifts, gene amplification, or other mechanisms, protooncogenes may be transformed into oncogenes that can lead to abnormal proliferation of cells or stimulate abnormal cell survival by interfering with apoptosis. At present, many studies have made a comprehensive assessment of the important role of oncogene in the development of ovarian cancer [25].

It has been reported that multidrug resistance of ovarian cancer may involve CXCR4, NRP1, ACO1, BDNF and HMGCR. 5 up-regulated genes and CDKN2C, FAS, SKP2, and 3 down regulated expression genes [26]. CXCR4 encoding chemokine receptor 4 (chemokine receptor 4), the receptor ligand stromal cell-derived factor -1 (stromal cell derived factor-1, SDF-1) specific binding, play an important role in tumor cell proliferation, invasion and migration, and to participate in the progression and prognosis of tumor. In the research of Kwong, CTCE-9908 (CXCR4 receptor antagonist) treatment and paclitaxel in patients with ovarian cancer, the results show that a combination of drugs can induce ovarian cancer cell mitosis disorder, increase cell toxicity, therefore concluded that CXCR4 is expected to become a new target for ovarian cancer therapy [27]. NRP1 is a receptor of vascular endothelial growth factor (VEGF), and it is a potential antitumor therapeutic target for many kinds of human malignant tumors. NRP1 can promote the self-renewal ability of cancer stem cells. Its high expression in cancer tissues will seriously affect the prognosis of patients, and it is of great significance in the development of ovarian cancer [28]. The over expression of NRP1 in cancer tissues seriously affects the prognosis of patients. It is a potential anti-cancer therapeutic target of human multiple malignancies including ovarian cancer $[29,30]$. Other studies have reported that AKT1, AKT2, BAX, EGFR, JUN, MYC, NFKB1, PIK3CA, RAS, and SRC are key regulators of ovarian cancer resistance. AKT and BAX is a downstream gene in a number of oncogene mediated ovarian cancer resistance pathways and plays a central role.

Multidrug resistance in ovarian cancer often involves many factors, such as signal transduction, metabolic pathways, protease pathways, and DNA damage and repair. Some signaling pathways such as MAPK, PI3K/AKT and BAX activate [31-33], or some signaling pathways such as P53 inactivation [34,35], which may also affect cell growth, 
proliferation, differentiation and apoptosis, and then form multidrug resistance of ovarian cancer. This article will briefly introduce and summarize these signal paths.

\section{MAPK}

Many studies have confirmed that MAPK signaling pathway is closely related to chemotherapeutic drug resistance in ovarian cancer. At present, the MAPK family has been found to include 8 subgroups, such as ERK, ERK3, ERK5/BMK1, ERK7, ERK8, JNK, NLK, P38 MAPK, etc. In epithelial ovarian cancer, the dose of platinum drugs is closely related to the continuous activation of ERK1/2 and JNK. The continuous activation of ERK1/2 and JNK can induce DNA damage and apoptosis. However, the continuous activation of ERK1/2 and JNK signals can also induce the production of platinum resistance in ovarian cancer cells. Conversely, the sensitivity of ovarian cancer cells to platinum drugs can be enhanced by inhibiting the continuous activation of ERK1/2 signals, [32,33]. It is also suggested that the activation of ERK1/2 pathway and the inactivation of the P38MAPK pathway can induce the resistance of ovarian cancer cells to platinum drugs. In addition, MAPK1, also known as ERK, is considered to be involved in [31] in the MAPK1 mediated MAPK signaling pathway associated with ovarian cancer resistance.

\section{PI3K/AKT}

The mechanism of drug resistance in ovarian cancer is to stimulate cell survival and block apoptosis. PI3K/AKT signaling pathway is the main pathway for cancer cells to survive. AKT can be activated by various stimuli, such as growth factors, protein kinase inhibitors, etc. For example, the expression of ACTN4 mRNA can increase the phosphorylation of AKT and stimulate AKT shift and cell proliferation. PTEN is a negative regulating factor of the PI3K/AKT pathway. The decrease of PTEN can also lead to the increase and activation of AKT phosphorylation, thereby promoting cell survival and proliferation, blocking cell apoptosis, and eventually leading to the occurrence of platinum resistance.

\section{BAX}

$\mathrm{BAX}$ is a cell death stimulator and homologous to anti apoptotic BCL2 protein. BAX and BCL2 or BCL2-like 1 (BCL-XL, also known as BCL2L1), can form heterozygous two polymers. BAX-mediated apoptosis depends on the relative amount of BAX, that is, whether BCL2 and/or BAX:BCL-XL heterodimers appear in cells. If heterodimers appear, the amount of BAX decreases. BAX-mediated apoptosis is reduced. The apoptosis pathway induced by BAX is a necessary pathway for chemotherapy induced cell death, so the down-regulation of BAX is closely related to chemotherapeutic drug resistance. Besides, $\mathrm{BAX}$ is also a downstream gene of multiple ovarian cancer resistance related genes. These results show the important role of BAX and AKT in the drug resistance of ovarian cancer [31].

\section{tp53}

P53 is a tumor suppressor gene involved in inducing cell cycle arrest, promoting apoptosis and DNA repair. Many studies have confirmed that P53 signaling pathway is associated with the pathogenesis and prognosis of ovarian cancer, and the sensitivity of platinum chemotherapy is closely related to the functional status of P53 $[36,37]$. The P53 gene regulatory network (P53 signaling pathway) is first proposed by Vogelstein, et al. in 2000. It is pointed out that cell life activity is regulated by gene interaction in P53 gene regulatory network. Among them, MDM2-P53-P21WAF1/CIPI is the most important pathway in the P53 signaling pathway, and any abnormal gene in this pathway may lead to the occurrence of tumor.

Apoptosis and phosphorylation are the two most important biological pathways of oncogene resistance to ovarian cancer [31]. Apoptosis is an inseparable part of the physiological balance, and the relationship between apoptosis and resistance depends on who is more dominant in the process of cell proliferation and differentiation. For example, the oncogene BCL2, a negative regulator of apoptosis, is a contribution to drug resistance by reducing apoptosis. DOK2 can induce carboplatin resistant in ovarian cancer by inhibiting apoptosis [36]. Phosphorylation and dephosphorylation can regulate the signal transduction of growth factors and cytokines, thereby regulating cell reproduction, differentiation, survival and apoptosis, and the occurrence and development of cancer [37]. The expression of many genes associated with ovarian cancer resistance is also regulated by phosphorylation, such as AKT, SRC, and STAT3[38,39]. In addition, the activation of other pathways, such as mismatch repair, can also induce the production of chemotherapeutic drug resistance in ovarian cancer.

\section{BRCA2}

BRCA2 gene is an oncogene. There is an important relationship between inhibition, carcinogenesis and prognosis is located in 13q1213, a group of ovarian cancer and breast cancer. The genomic DNA is about $70 \mathrm{~kb}$, encoding $10987 \mathrm{bp}$, and has 27 exons. The eleventh exon is the largest exon. It is the site of binding with DNA repair related protein 51 (DNA damage repair protein 51, RAD 51). The mRNA of BRCA2 is about $10 \mathrm{~kb}$ long and encodes a tumor-inhibitory BRCA2 protein consisting of 3418 amino acid residues.

BRCA2 is closely related to RAD51 in homologous recombination [40] and DNA damage repair function together. At the end of the carboxyl group of BRCA2, there is a domain called the BRCA2 DBD (DNA/DSS1-binding domain) domain, which contains 5 domains similar to the DNA binding motifs: Screw, OB1, OB2, OB3 domain and domain tower. When the BRCA2 mutation in the tumor, 5 missense domain mutation incidence rates were $25 \%, 25.4 \%, 8.6 \%$, $8.6 \%$ and $32.4 \%$. When the DNA double strand damage, DNA damage will send a signal, the signal will be further activated such as ataxia telangiectasia gene (ataxia-telangiectasiamutated, ATM), antithrombin (antithrombin receptor, ATR) receptor like kinase activity in the catalytic BRCA2/RAD51 phosphorylation of protein complexes [8]. The transformation from inactive form into an active form, and then, the BRCA2 protein carrying RAD51 protein transport to the common double strand DNA damage sites involved in the reorganization of double strand DNA damage repair, play the role of tumor suppressor.

BRCA2 mutant cells have homologous recombination function defects, and DNA damage can also cause insufficient aggregation of RAD51 nuclei. DNA repair defects can produce another result, which is highly sensitive to the widely used anticancer drug DNA injuring agent. DNA damaging agents have a prominent role in DNA repairdefective tumor cells [41]. BRCA2 mutation in patients with ovarian cancer were sensitive to platinum, but at the end of this part of patients will still become platinum resistance.

The present study suggests that the Second mutation of some tumor suppressor genes carrying primary tumor may be related to the acquired platinum resistance, such as TP53, breast cancer susceptibility gene 1 and 2 (BRCA1 and BRCA2), such as TP53, breast cancer susceptibility genes 1 and 2 (BRCA1 and BRCA2). Studies have shown that in BRCA1 
and BRCA2 ovarian cancer carrying the reading frame shift mutation, the acquired platinum resistance is produced by two mutations in the gene. Corrected the Open Reading Frames (ORF) of mutant BRCA1/2, restored the expression of wild type BRCA1/2 and DNA homologous recombination repair function, which led to the occurrence of acquired platinum resistance in ovarian cancer. In the cell line of pancreatic cancer or ovarian cancer with BRCA2 mutation, the two mutation of BRCA2 can be induced by cisplatin or polyadenosine two phosphate ribose polymerase (poly ADP-ribose polymerase (PARP) inhibitor) [42]. As a result of this reverting mutation, the truncated BRCA2 protein is renewed and carries the function of homologous recombination. So, the BRCA2 mutation occurred two times the use of platinum drugs in BRCA2 mutant tumors is acquired. The mechanism of resistance to platinum.

BRCA2 makes DNA damage repair function missing, and cell genetic instability increases the incidence of ovarian cancer, and ovarian cancer is sensitive to platinum based DNA damage agents, after the two mutation of BRCA2, in the BRCA2 ovarian cancer carrying frameshift mutation, corrected the open reading frame of the mutant BRCA2, restored the expression of wild type BRCA2 and DNA homologous recombination repair function, which led to the occurrence of acquired platinum resistance in ovarian cancer. However, the current BRCA2 two gene mutation induced resistance in ovarian cancer is not very clear.

In summary, chemotherapy is an important treatment for patients with ovarian cancer. Although the initial ovarian cancer patients curative effect is high, studies have pointed out that after paclitaxel and platinum drugs such as first-line chemotherapy in patients with advanced initial reaction rate as high as $80 \%$, the complete remission rate of $40 \% \sim 60 \%$, but the majority of patients will relapse and death, the data showed that $70 \% \sim 80 \%$ patients with recurrence, 5 years survival rate is still around $30 \% \sim 45 \%$. The main factor which leads to the failure of chemotherapy and the improvement of survival rate is the emergence of chemotherapeutic drug resistance [26]. Therefore, it is an urgent problem to find an effective way to solve the drug resistance of chemotherapy.

Using NGS detection technique, the tumor specific molecular changes can be detected in the blood circulation, and the whole genome information of tumor can be detected in real time and dynamically. Therefore, it has broad application prospects in exploring the molecular mechanisms of tumor recurrence, metastasis and drug resistance. The emergence of this technology is in line with the current trend of "precise medical care" in the current tumor. Therefore, the molecular mechanism of ctDNA analysis of the drug resistance of ovarian cancer by NGS technique may open a new situation for the treatment of patients with recurrent ovarian cancer.

\section{References}

1. Diaz LA Jr, Bardelli A (2014) Liquid biopsies: genotyping circulating tumor DNA. $J$ Clin Oncol 32: 579-586. [Crossref]

2. Gerlinger M, Rowan AJ, Horswell S (2012) Intratumor heterogeneity and branched evolution revealed by multiregion sequencing. $N$ Engl J Med 366: 883-892.

3. Vogelstein B, Papadopoulos N, Velculescu VE, Zhou S, Diaz LA Jr, et al. (2013) Cancer genome landscapes. Science 339: 1546-1558. [Crossref]

4. Diaz LA Jr, Williams RT, Wu J (2012) The molecular evolution ofacquired resistance to targeted EGFR blockade in colorectal cancers[J]. Nature 486: 537-540.

5. Overman MJ, Modak J, Kopetz S, Murthy R, Yao JC, et al. (2013) Use of research biopsies in clinical trials: are risks and benefits adequately discussed? J Clin Oncol 31: 17-22. [Crossref]
6. Murtaza M, Dawson SJ, Tsui DW, Gale D, Forshew T, et al. (2013) Non-invasive analysis of acquired resistance to cancer therapy by sequencing of plasma DNA. Nature 497: 108-112. [Crossref]

7. De Mattos-Arruda L, Cortes J, Santarpia L, Vivancos A, Tabernero J, et al. (2013) Circulating tumour cells and cell-free DNA as tools for managing breast cancer. Nat Rev Clin Oncol 10: 377-389. [Crossref]

8. Diaz LA Jr, Williams RT, Wu J (2012) The molecular evolution of acquired resistance to targeted EGFR blockade in colorectal cancers. Nature 486: 537-540.

9. Forshew T, Murtaza M, Parkinson C, Gale D, Tsui DW, et al. (2012) Noninvasive identification and monitoring of cancer mutations by targeted deep sequencing of plasma DNA. Sci Transl Med 4: 136ra68. [Crossref]

10. Dawson SJ, Tsui DW, Murtaza M, Biggs H, Rueda OM, et al. (2013) Analysis of circulating tumor DNA to monitor metastatic breast cancer. N Engl J Med 368: 11991209. [Crossref]

11. Higgins MJ, Jelovac D, Barnathan E, et al. Detection of tumor PIK3CA status in metastatic breast cancer using peripheral blood. Clin Cancer Res 18: 3462-3469.

12. Vogelstein B, Papadopoulos N, Velculescu VE, Zhou S, Diaz LA Jr, et al. (2013) Cancer genome landscapes. Science 339: 1546-1558. [Crossref]

13. Chetan Bettegowda, Mark Sausen, Rebecca J. Leary (2014) Detection of Circulating Tumor DNA in Early- and Late-Stage Human Malignancies. Sci Transl Med 6: 224ra24.

14. Madhavan D, Wallwiener M, Bents K (2014) Plasma DNA integrityas a biomarker for primary and metastatic breast cancer andpotential marker for early diagnosis[J]. Breast Cancer Res Treat 146: 163-174.

15. da Silva Filho BF, Gurgel AP, Neto MÁ, de Azevedo DA, de Freitas AC, et al. (2013) Circulating cell-free DNA in serum as a biomarker of colorectal cancer. J Clin Pathol 66: 775-778. [Crossref]

16. Leszinski G, Lehner J, Gezer U, Holdenrieder S (2014) Increased DNA integrity in colorectal cancer. In Vivo 28: 299-303. [Crossref]

17. Kim K, Shin DG, Park MK (2014) Circulating cell-free DNA as apromising biomarker in patients with gastric cancer: diagnosticvalidity and significant reduction of cfDNA after surgical resection[J]. Ann Surg Treat Res 83: 136-142.

18. Spindler KL, Pallisgaard N, Vogelius I (2012) Quantitative cell-freeDNA, KRAS, and BRAF mutations in plasma from patients withmetastatic colorectal cancer during treatment with cetuximab andirinotecan[J]. Clin Cancer Res 18: 1177-1185.

19. Dawson SJ, Tsui DW, Murtaza M, Biggs H, Rueda OM, et al. (2013) Analysis of circulating tumor DNA to monitor metastatic breast cancer. $N$ Engl J Med 368: 11991209. [Crossref]

20. Forshew T, Murtaza M, Parkinson C (2012) Noninvasive identificationand monitoring of cancer mutations by targeted deep sequencing ofplasma DNA[J]. Sci Transl Med 4: $136 \mathrm{ra} 168$.

21. Diaz LA Jr, Williams RT, Wu J, et al. The molecular evolution ofacquired resistance to targeted EGFR blockade in colorectal cancers[J]. Nature 486(7404): 537-540.

22. Song Xu, Feng Lou, YiWu (2016) Circulating tumor DNA identified by targeted sequencing inadvanced-stage non-small cell lung cancer patients. Cancer Letters 370: 324-331.

23. Evelyn Kidess, Kyra Heirich, Matthew Wiggin, Valentina Vysotsk (2014) Mutation profiling of tumor DNA from plasma and tumor tissue of colorectal cancer patients with a novel, high-sensitivity multiplexed mutation detection platform. Oncotarget 6 : 2549-2561

24. Tang JC, Feng YL, Guo T, Xie AY, Cai XJ (2016) Circulating tumor DNA in hepatocellular carcinoma: trends and challenges. Cell Biosci 6: 32. [Crossref]

25. Zhao M, Sun J, Zhao Z (2012) Distinct and competitive regulatory patterns of tumor suppressor genes and oncogenes in ovarian cancer. PLoS One 7: e44175. [Crossref]

26. CHEN Changxian, HU Yanling, LI Li (2016) Bioinformatics Analysis of Genes Related to Multidrug Resistance in Ovarian Cancer. Cancer Res Prev Treat 43: 492-496.

27. Ray P, Lewin SA, Mihalko LA, Schmidt BT, Luker KE, et al. (2011) Noninvasive imaging reveals inhibition of ovarian cancer by targeting CXCL12-CXCR4. Neoplasia 13: 1152-1161. [Crossref]

28. Beck B, Driessens G, Goossens S, Youssef KK, Kuchnio A, et al. (2011) A vascular niche and a VEGF-Nrp1 loop regulate the initiation and stemness of skin tumours Nature 478: 399-403. [Crossref]

29. Grandclement C, Borg C (2011) Neuropilins: a new target for cancer therapy. Cancer (Basel) 3: 1899-1928. [Crossref] 
Du Z (2018) The research progress of application of sequencing technology to analyze the genetic information of malignant tumor, especially drug resistant recurrent ovarian cancer

30. Siamakpour-Reihani S, Owzar K, Jiang C (2015) Prognosticsignificance of differential expression of angiogenic genes inwomen with high-grade serous ovarian carcinoma[J]. GynecolOncol 139: 23-29.

31. Liu X, Gao Y, Lu Y, Zhang J, Li L, et al. (2015) Oncogenes associated with drug resistance in ovarian cancer. J Cancer Res Clin Oncol 141: 381-395. [Crossref]

32. Rosanò L, Cianfrocca R, Spinella F (2011) Acquisition ofchemoresistance and EMT phenotype is linked with activation ofthe endothelin A receptor pathway in ovarian carcinoma cells[J]. Clin Cancer Res 17: 2350-60.

33. Masoumi-Moghaddam S, Amini A, Wei AQ (2015) Sprouty2protein in prediction of post-treatment ascites in epithelial ovariancancer treated with adjuvant carbotaxol chemotherapy[J]. Am J Cancer Res 5: 2498-507.

34. Yang-Hartwich Y, Soteras MG, Lin ZP, Holmberg J, Sumi N, et al. (2015) p53 protein aggregation promotes platinum resistance in ovarian cancer. Oncogene 34: 3605-3616. [Crossref]

35. Woo MG, Xue K, Liu J (2012) Calpain-mediated processing ofp53-associated parkinlike cytoplasmic protein (PARC) affectschemosensitivity of human ovarian cancer cells by promoting p53subcellular trafficking[J]. J Biol Chem 287: 3963-3975.
36. Lum E, Vigliotti M, Banerjee N, Cutter N, Wrzeszczynski KO, et al. (2013) Loss of DOK2 induces carboplatin resistance in ovarian cancervia suppression of apoptosis. Gynecol Oncol 130: 369-376.

37. Acosta KB, Tibolla MM, Tiscornia MM, Lorenzati MA, Zapata PD (2011) Recent patents related to phosphorylation signalingpathway on cancer. Recent Pat DNA Gene Seq 5: $175-184$

38. Ji T, Gong D, Han Z, Wei X, Yan Y, et al. (2013) Abrogationof constitutive Stat3 activity circumvents cisplatin resistantovarian cancer. Cancer Lett 341: 231-239

39. Su L, Wang J, Tao Y, Shao X, Ding Y, et al. (2015) BRCA2 N372H Polymorphism and Risk of Epithelial Ovarian Cancer: An Updated Meta-Analysis With 2344 Cases and 9672 Controls. Medicine (Baltimore) 94: e1695. [Crossref]

40. Diamond JR, Borges VF, Eckhardt SG, Jimeno A (2009) BRCA in breast cancer: from risk assessment to therapeutic prediction. Drug News Perspect 22: 603-608. [Crossref]

41. Evers B, Helleday T,Jonkers J (2010) Targeting homologous recombination repair defects in cancer[J]. Trends Pharmacol Sci 31: 72-80.

42. Sakai W, Swisher EM, Jacquemont C (2009) Functional restoration of BRCA2 protein by secondary BRCA2 mutations in BRCA2-mutated ovarian carcinoma[J]. Cancer Res 69 :6381- 6386.

Copyright: (C2018 Du Z. This is an open-access article distributed under the terms of the Creative Commons Attribution License, which permits unrestricted use, distribution, and reproduction in any medium, provided the original author and source are credited. 